\title{
Pour accueillir la décentralisation : une MRC transformée, plus démocratique et plus efficace
}

\author{
Yvon Leclerc \\ Journal Réseau
}

Au tournant des années 1980, deux mesures importantes ont été prises, presque simultanément, pour engager le monde municipal sur la voie du $21^{\mathrm{e}}$ siècle.

\section{La loi 125}

Tout d'abord, le législateur a réuni dans une même structure territoriale la Loi des cités et villes et le Code municipal en créant les Municipalités régionales de comté (MRC), dont le découpage était calqué, à peu de chose près, sur les limites territoriales des conseils de comté. C'est la Loi sur l'aménagement et l'urbanisme ou loi 125 qui confiait à la MRC le mandat d'élaborer un schéma d'aménagement du territoire. Ce geste provoqua une petite révolution locale car, depuis plus d'un siècle, le monde municipal était divisé en municipalités de 5000 habitants et plus et en

\section{Les MRC sont progressivement passées de l'apprivoisement à la concertation, puis à une mission qui s'apparente de plus en plus au développement.} tant réglée. fiscalité québécoise confiait au monde municipal la quasi-exclusivité du champ foncier qu'il partageait auparavant avec les commissions scolaires. Cette décision a eu le mérite de clarifier les champs fiscaux mais, 25 ans plus tard, la question n'est pas pour au-

Ces municipalités peuvent-elles faire face à leurs responsabilités d'aujourd'hui avec les ressources fiscales qui leur avaient été confiées à cette époque ? Peuventelles accueillir des responsabilités nouvelles qui marqueraient une importante reconfiguration de l'État québécois, qu'il soit devenu souverain ou non ? Et où atterriraient ces nouvelles responsabilités ? À la municipalité ou à la MRC? Sachant que les deux Unions municipales insistent pour que les nouvelles responsabilités découlant de la décentralisation devraient municipalités de 5000 habitants et moins, les charges étant différentes pour les unes et les autres. Et surtout, les unes et les autres n'étaient pas habituées à travailler ensemble. Le schéma d'aménagement avait donc une mission d'apprivoisement des unes et des autres. Cet apprivoisement a été long et difficile dans certains milieux. Depuis, les MRC en sont à leur deuxième et même troisième édition du schéma, et le législateur leur a confié de nouvelles missions en matière d'environnement et de développement local. Avec le résultat que les MRC sont progressivement passées de l'apprivoisement à la concertation, puis à une mission qui s'apparente de plus en plus au développement. Avec en prime une esquisse de vision régionale.

\section{La réforme de la fiscalité}

L'autre mesure importante concerne le revenu des municipalités. En 1979, une importante réforme de la être confiées à des structures imputables, la MRC, dans sa constitution actuelle, ne répond pas à cette exigence.

\section{Le territoire du développement}

Pourtant, l'État québécois a tranché en 1996 : le territoire de la concertation resterait la région administrative et le territoire du développement serait celui de la MRC. C'est le lieu privilégié des solidarités entre les habitants d'un territoire, le territoire d'appartenance. L'évolution des quinze dernières années a confirmé ce choix. La création des Centres locaux d'emploi (CLE), la mise en place des Centre locaux de développement (CLD) et des Centres locaux de services communautaires (CLSC) ainsi que la réforme des territoires des commissions scolaires ont respecté cette décision. Le gouvernement a donc officialisé, à plusieurs reprises, la MRC comme le territoire des acteurs du 
développement et des services aux personnes dans l'architecture de ses politiques publiques.

Mais avant de s'arrêter aux structures d'accueil de la décentralisation qui permettrait aux régions de s'outiller convenablement pour assurer leur développement, il convient de s'interroger sur la capacité actuelle des MRC à répondre à l'accueil de nouvelles responsabilités.

\section{Deux préalables à considérer}

Tout d'abord, depuis la Révolution tranquille, l'urbanisation du Québec s'est poursuivie à vive allure et s'est développée sur la base d'un modèle polycentrique. Ce phénomène est visible à l'œil nu : on relève environ 35 centres urbains, distribués sur l'ensemble du territoire, qui rassemblent près de $90 \%$ de la population. La croissance de la population et le dynamisme des municipalités rurales sont la plupart du temps proportionnels à leur proximité des centres. Et n'eut été des pactes ruraux de la Politique nationale de la ruralité, le situation serait pire encore.

L'autre considération à prendre en compte concerne le fait que la richesse se crée largement dans les villes. Cette observation a été bien documentée par Jane Jacobs dans ses livres.

Ceci dit, voici trois exemples de dysfonction qui démontrent que la structure politique de la MRC devra être revue et corrigée avant d'aller de l'avant avec la décentralisation de nouvelles responsabilités.

\section{Trois exemples de dysfonction}

\section{Les structures municipales actuelles entretiennent et creusent les inégalités entre les citoyens et les municipalités d'un même territoire de MRC}

Plusieurs cas pourraient être utilisés pour illustrer ce phénomène; examinons celui de Gaspé et de la MRC La Côte-de-Gaspé. On se rappelle que la ville de Gaspé est née de la fusion de douze municipalités entourant la ville-centre de Gaspé à l'occasion de la création du parc Forillon en 1970. Cette fusion, bâclée en six semaines, a fait de Gaspé la troisième ville la plus étendue du Canada. Une dizaine d'années plus tard, au moment de tracer les limites territoriales des MRC, la population de quatre municipalités riveraines de Gaspé, soit Cloridorme, Petite Vallée, Grande
Vallée et Murdochville, ont choisi de se joindre à la ville de Gaspé pour former la MRC La Côte-de-Gaspé. La ville de Gaspé compte pour $80 \%$ de la population de la MRC.

Or, après avoir connu une diminution importante entre 1996 et 2001, l'exode s'est arrêté entre 2001 et 2006. Et pour cause. La ville a largement su tirer profit de l'éolien (une usine de pales et une deuxième en implantation) et du choix de Desjardins d'y installer son centre de traitement des prêts étudiants.

Entre 2004 et 2007, la richesse foncière uniformisée est passée de $508 \mathrm{M} \$$ à plus de $633 \mathrm{M} \$$. Entre 2006 et 2007, la RFU de la ville de Gaspé a bondi de $67 \mathrm{M}$, soit plus de quinze fois celle des quatre autres municipalités de la MRC prises ensemble. Et l'enrichissement de la ville de Gaspé se poursuit.

\section{Il convient de s'interroger sur la capacité actuelle des MRC à répondre à l'accueil de nouvelles responsabilités.}

Côté habitation, la ville de Gaspé connaît un taux d'inoccupation de moins de $1 \%$, au point qu'il est devenu très difficile de se loger au centre-ville. La valeur des maisons augmente, les commerces y font de bonnes affaires, les services scolaires, municipaux, de santé y sont de bonne qualité. Et évidemment, les recettes fiscales de la ville suivent.

Constat : la richesse produite par la ville de Gaspé ne profite qu'indirectement aux contribuables vivant dans les quatre municipalités du territoire. Du reste, si la Ville facturait tous les services offerts à ces municipalités, l'écart se creuserait encore davantage. Pourquoi la richesse produite par le principal pôle de développement de la MRC ne profiterait-elle pas aux habitants des autres municipalités du territoire d'appartenance? L'autonomie municipale vaut-elle ce prix ? Les maires de la MRC La Côte-de-Gaspé l'ont compris et ont entrepris une démarche d'analyse coûtsbénéfices des fonctions de la MRC dont certaines pourraient être assumées par la ville de Gaspé, quitte à réserver des fonctions plus politiques à la MRC.

En somme, ces maires réalisent que la structure actuelle de la MRC, sa mission et son mécanisme de redistribution de la richesse doivent être revus en fonction des responsabilités d'aujourd'hui si on veut éviter 
de créer ou de creuser des écarts entre les municipalités et les citoyens qui les habitent. De telles dysfonctions peuvent être signalées dans un grand nombre de $\mathrm{MRC}$; une recension rigoureuse ferait apparaître ces inégalités de façon éclatante. (Voilà un beau sujet de recherche!)

Ce n'est pas la première fois qu'on constate l'iniquité qui existe entre les municipalités; les couples villageparoisse en constituent des cas souvent dénoncés. De même que les villes qui abritent des usines de grande envergure et qui encaissent des recettes foncières importantes sans obligation de partage régional. Il faut reconnaître, toutefois, que plusieurs de ces villes ne se font pas prier pour offrir des services régionaux sans compensation financière. Mais cela reste aléatoire, relevant de la bonne volonté du maire de la ville principale, en l'absence de mécanisme institutionnel de redistribution. Et prouve que l'État québécois ne peut s'en remettre qu'à la progressivité de l'impôt sur le revenu pour assurer la redistribution de la richesse.

\section{Vingt-sept ans plus tard, le rêve du pays est toujours d'actualité et la décentralisation est gardée au frais.}

\section{Le processus de décision et le grand nombre de municipalités dans une même MRC rendent difficile une vision cohérente de l'aménagement et de développement d'un territoire}

Rappelons que la réforme de la fiscalité municipale du tournant des années 1980 a laissé tout le champ de l'impôt foncier aux municipalités. Avec comme conséquence que si une municipalité veut augmenter ses revenus, elle doit augmenter ses périmètres d'urbanisation voués à la construction domiciliaire ou industrielle. Dans les zones périurbaines comme la Côtede-Beaupré, cette pression fiscale peut conduire à la destruction des paysages et à une agression envers l'agriculture sous toutes ses formes. Voyons la situation d'un peu plus près.

La Côte-de-Beaupré, qui fait maintenant partie de la Communauté métropolitaine de Québec, a vu sa responsabilité de l'aménagement passer à cette nouvelle instance née de la fusion de 2002. Or, dans son chantier de planification stratégique pour les 25 prochaines années, la Communauté métropolitaine a consenti une provision de 50 hectares pour la construction ré- sidentielle et industrielle. Par un calcul alambiqué fait de régularisation d'états de fait et de nouveaux empiètements en zone agricole, les 8 municipalités composant la MRC ont prévu 28 hectares de prédation dans les terres agricoles.

Mais c'est encore beaucoup trop! Car ces empiètements se produisent dans les plus belles terres de la vallée du Saint-Laurent, celles que Champlain appelait, dès son premier voyage, les Beaux Prés. Sans compter qu'en zone maraîchère, 28 ha en culture peuvent créer plusieurs emplois. Le comble, c'est que la seule municipalité de Beaupré dispose déjà de plus de 50 hectares pour des fins domiciliaires. Et Boischatel, compte encore de nombreux terrains à bâtir en dehors de la zone agricole. Ce qui revient à dire que ces empiètements en zone agricole sont totalement inutiles et que les paysages seront irrémédiablement gaspillés.

On peut avancer que si les huit municipalités de la Côte-de-Beaupré étaient regroupées en une seule ville plutôt qu'en MRC, la question ne se poserait même pas. Les élus de la ville pourraient se consacrer à un aménagement respectueux de la vocation historique, patrimoniale et culturelle. Et ce, sans chercher à étendre les périmètres d'urbanisation pour augmenter la colonne des revenus de leur budget et sans dénaturer les paysages champêtres qui font partie de l'identité de la Côte-de-Beaupré.

\section{L'organisation municipale actuelle constitue un obstacle à la décentralisation}

La dernière fois qu'un gouvernement a envisagé de décentraliser des responsabilités importantes vers le monde municipal remonte aux discussions entourant la Loi sur l'aménagement et l'urbanisme, dans le célèbre Fascicule V, qui n'a jamais vu le jour, mais que les aménagistes connaissent bien. Dans ce document, le gouvernement songeait à créer une centaine de territoires appelés les Municipalités régionales de comté à qui seraient confiées plusieurs nouvelles responsabilités si la population lui accordait le mandat de réaliser la souveraineté-association. Vingt-sept ans plus tard, le rêve du pays est toujours d'actualité et la décentralisation est gardée au frais, alors que le monde municipal et les MRC trépignent d'impatience à l'idée de participer plus activement à la mise en valeur économique, sociale et culturelle de leur milieu.

Quant au cadre légal de la MRC, il n'a guère évolué. La Loi sur l'aménagement et l'urbanisme a connu des 
amendements à la marge, alors que la situation actuelle mérite une nouvelle construction juridique adaptée au contexte d'aujourd'hui. Concerter un milieu autour d'un schéma d'aménagement ne fait pas appel aux mêmes pratiques administratives que la gestion managériale de projets de développement. Et la formule actuelle de démocratie par délégation de la MRC n'a pas suivi l'évolution des nouvelles pratiques.

Les deux Unions municipales s'entendent sur ce point : la décentralisation se réalisera auprès d'instances municipales directement imputables envers la population ou elle ne se fera pas. Et la constitution actuelle de la MRC ne répond pas à cette condition. Or, rappelons que le Québec compte environ 35 municipalités regroupant $90 \%$ de la population et 116 municipalités de 3000 à 5000 habitants qui jouent un rôle de pôle régional. N'est-ce pas en examinant cette réalité qu'il faut commencer?

\section{« Les résidents des municipalités rurales peu populeuses ont tendance à reporter sur les épaules du CLD la responsabilité du développement économique local. »}

\section{S’appuyer sur des solidarités actives}

Jacques Brisebois était jusqu'à tout récemment viceprésident de la Commission municipale du Québec. Il fut auparavant maire de Mont-Laurier, préfet de la MRC d'Antoine-Labelle et président du CLD. Il vient d'accepter de retourner à la pratique à Saint-FaustinLac-Carré. Son expérience l'amène à appuyer l'architecture de l'organisation municipale sur « des solidarités actives », sur l'importance des liens entre les individus et les groupes qui composent les communautés locales et sur les relations qui existent entre les municipalités de petite taille et les services locaux et régionaux susceptibles de toucher la vie quotidienne des citoyens et citoyennes de la MRC.

Dans une longue lettre qu'il nous adressait au sujet de la décentralisation, Jacques Brisebois sert une mise en garde sur les effets de la concentration de certains services publics à l'échelle de la MRC, phénomène qui peut entraîner une forme de déresponsabilisation des citoyens lorsque leur engagement est nécessaire. Il convient volontiers que tous les services publics exigent une forme d'engagement responsable des ci- toyens. Il faut cependant distinguer les services de proximité liés à la propriété qui s'adressent au civisme des personnes de ceux qui exigent un engagement des individus, comme par exemple les activités de développement de leur milieu. «L'effet de la concentration des services au palier de la MRC comme d'ailleurs dans la ville-centre - ou la municipalité centre de services qui en tient lieu - pourrait avoir un effet démobilisateur sur l'engagement des personnes ».

Par exemple, tout en étant d'accord avec l'arrivée des CLD, il craint un effet pervers, celui de la déresponsabilisation locale à l'égard du développement. « Les résidents des municipalités rurales peu populeuses ont tendance à reporter sur les épaules du CLD la responsabilité du développement économique local. Le CLD va s'occuper de ça! peut-on déjà entendre. Et elles n'ont pas tort puisqu'elles contribuent au financement du CLD et que le mandat du CLD concerne l'ensemble du territoire de la MRC. »

\section{Le renforcement des institutions municipales}

Pour éviter ce piège de la déresponsabilisation, Jacques Brisebois propose le renforcement des institutions municipales locales. " Dans une MRC à grande étendue, qui compte un centre et quelques sous-centres, comme la MRC d'Antoine-Labelle, le renforcement des centres de services signifie également un renforcement de la MRC. Ainsi, passer de 22 à 4 ou 5 municipalités devrait permettre, d'une part, une meilleure prise sur les services à rendre localement et faciliter, d'autre part, la gestion régionale. Regrouper les municipalités rurales peu populeuses sur la base des zones d'influence réintroduirait cette capacité de se responsabiliser et d'accompagner les milieux plus éloignés. »

Ce qui compte en définitive, c'est de se recentrer sur la question de base : que vise le renforcement des institutions locales? Des économies d'échelle? Une livraison de services de meilleure qualité ? L'atteinte d'une meilleure équité fiscale? L'accompagnement des petites communautés ? "Par rapport au discours de l'éloignement et de l'étalement, le premier objectif du renforcement, selon lui, devrait être l'accompagnement des municipalités éloignées qui n'ont pas une masse critique suffisante pour faire face à certaines responsabilités. Ce faisant, il ne faudrait pas rendre inopérantes celles qui sont en mesure d'y faire face. 
Au contraire, il faut procéder au renforcement local en réorganisant les zones d'influence. »

\section{La définition des zones d'influence}

Comment définir les zones d'influence? Les villescentres sont à proprement parler des pivots de zones d'influence. Montréal comme métropole du Québec étend sa zone d'influence sur l'ensemble du Québec, en ce sens qu'aucune région n'échappe, d'une façon ou d'une autre, à son attraction. Au-delà de ces considérations globales, sa zone d'influence immédiate est la région métropolitaine, telle que définie par la Communauté métropolitaine de Montréal (CMM). Il en est ainsi pour chacune des villes dont on peut reconnaître, de façon évidente, qu'elles occupent sur une base d'agglomération un espace économique, social et culturel. Naturellement, un grand nombre de services et une large part des investissements industriels s'y retrouvent.

En dehors des MRC où sont les régions métropolitaines de recensement (villes de 100000 habitants et plus) et les agglomérations de recensement (villes de 10000 à 100000 habitants), il y a une cinquantaine de MRC où il n'y a pas d'agglomération urbaine au sens strict de la définition statistique. Nonobstant ce fait, bon nombre de ces municipalités exercent des fonctions de centralité.

« C'est autour de celles-ci, dit Jacques Brisebois, que pourrait se mettre en place une politique de la ruralité en favorisant le développement, sous toutes ses formes, de zones d'influence efficaces et mobilisatrices. »

\section{L'organisation de villes-pivots responsables}

Une question se pose à propos d'une politique de renforcement des municipalités locales autour de l'idée des zones d'influence: quelle taille doit avoir une communauté pour être en mesure de rendre le maximum de services, le plus près du citoyen ?

" On remarque aisément, répond Jacques Brisebois, que dans une municipalité de moins de 500 habitants, il est de plus en plus difficile de conserver une école, une épicerie, une station d'essence, un service incendie, etc. C'est donc dire que les gens de ces municipalités doivent se déplacer pour leurs services de proxi- mité. Où vont-ils chercher ces services ? C'est une question importante et intéressante $»$.

« Dès que l'on rencontre une municipalité de 2000 ou 2500 habitants, on se rend compte que non seulement la population résidente y retrouve une bonne partie des services dont elle a besoin, mais que des non-résidents viennent également y chercher une partie des services non disponibles dans leur localité. Ces municipalités ont donc un rayonnement qui pourrait, s'il était mieux organisé, devenir le pivot d'une zone d'influence. »

«C'est un peu à l'image d'une poupée gigogne : l'offre des services part du plus petit au plus grand. Mais peut-on améliorer le premier niveau pour le rendre plus opérant en conservant les services le plus près des citoyens tout en se donnant une meilleure répartition des ressources, ainsi que de nouveaux et de meilleurs moyens pour accompagner le développement? »

La réflexion de Jacques Brisebois autour d'une organisation municipale appuyée sur des solidarités actives ne manque pas d'intérêt. Elle doit être prise en compte dans la nouvelle construction juridique de la $\mathrm{MRC}$, préalable nécessaire à la décentralisation. Réflexion particulièrement féconde pour les MRC rurales privées de ville-centre comme Drummondville, Rimouski ou Gaspé.

Les villes-centres sont à proprement parler des pivots de zones d'influence.

\section{Le territoire de la décentralisation et sa représentation politique}

Dans les faits, le territoire de la MRC constitue un choix logique et largement accepté pour accueillir la décentralisation; reste à rendre cette instance politiquement imputable et donc élue au suffrage universel. En pareil cas, on pourrait rencontrer deux types de MRC directement imputables devant la population. D'une part, des MRC rurales composées de quatre ou cinq villes-pôles présidées par un préfet élu au suffrage universel - comme le réclame la Fédération québécoise des municipalités. Et, d'autre part, des villesMRC qui répondraient aux problèmes d'organisation soulevés par les MRC où l'on trouve une ville importante rassemblant plus de la moitié de la population de la MRC. 
Conserver les municipalités locales dans leur forme actuelle équivaudrait à ignorer l'expérience des communautés urbaines qui a conduit à la constitution des villes de Québec, Montréal et Gatineau. Un seul exemple : la Communauté urbaine de Québec. Gérer l'aménagement du territoire et le développement économique, social et culturel à treize joueurs était devenu cauchemardesque: Sainte-Foy ne voulait pas de logements sociaux (les pauvres, c'est pour Québec), Vanier n'investissait pas un sou en développement économique (pourquoi l'aurait-elle fait puisque Québec le faisait et qu'elle y trouvait son compte sans débourser un sou!), Sillery achetait l'eau de la ville de Québec au prix coûtant et la revendait à profit à l'Université Laval, etc. Bref, une litanie d'horreurs qui a mené à la création d'une seule ville issue des treize villes antérieures.

Il est loin d'être évident que la situation se présenterait autrement si le choix d'élire les MRC au suffrage universel devait se doubler du maintien des municipalités locales dans leur statut actuel. L'arrivée de l'élection du préfet élu au suffrage universel confirme une tendance, certes, mais le préfet ne peut, à lui seul, et sans moyens autres que la légitimité démocratique, gérer le développement du territoire et arbitrer les décisions des élus municipaux qui eux, disposent des budgets d'opération.

Le choix de la ville-MRC et de MRC composées de villes-pôles pour aménager et construire les territoires semble donc une voie qui permettrait à la fois une meilleure répartition de la richesse produite par l'impôt foncier, une meilleure cohérence dans la gouvernance et un meilleur véhicule pour accueillir les mandats et responsabilités décentralisées. Et recomposer, localement, l'administration en silo de l'État. Suivant ce modèle, le Québec serait composé d'une centaine de territoires municipaux. Reste quelques questions.

\section{Le sentiment d'appartenance}

Comment distinguer la mission régionale des services de proximité et comment utiliser le puissant levier de mobilisation que constitue le sentiment d'appartenance ? Jusqu'ici, reconnaissons que le processus de fusion municipale a plutôt cherché à éteindre qu'à mettre en valeur le sentiment d'appartenance. Pourtant, c'est par le renforcement de ce sentiment d'appartenance par la mise en place de conseils de quartier dotés de réels pouvoirs et de moyens financiers que la solution se trouve. À la ville de Gaspé, on a entrepris de valoriser le sentiment d'appartenance aux municipalités fusionnées il y a 35 ans. De grandes affiches ont été placées à l'entrée des territoires et des lieuxdits pour les identifier. Excellente initiative qui vient corriger la tentative d'assimilation réalisée par la fusion de 1971.

Il faudra cependant faire plus. À Québec, par exemple, la Ville a mis sur pied des conseils de quartier dotés de pouvoirs et de moyens financiers significatifs. Ces conseils sont formés de bénévoles qui travaillent en lien (et parfois en opposition) avec les conseillers municipaux. Lorsque le maire Jean-Paul Lallier a quitté sa fonction, on comptait cinq conseils de quartier; trois ans plus tard on en dénombre 36 ! Et pourtant, les fonctions de ces conseils sont purement consultatives! On pourrait avancer deux raisons à cette progression rapide. D'abord, les conseils de quartier ont une mission claire et bien affirmée qui concerne des besoins très proches des contribuables et, ensuite, les citoyens sont bien informés et réagissent rapidement lorsqu'on touche à leur qualité de vie. Et puisqu'on y est, pourquoi ne pas explorer avec eux la voie du budget participatif tel que pratiqué à Porto Allegre?

On pourrait citer de nombreux exemples qui démontrent que le sentiment d'appartenance à un territoire constitue le fondement des politiques communautaires. Le lien qui unit l'individu à son lieu de naissance et à celui où il a vécu son enfance est une affaire de cœur.

\section{Le préfet ne peut, à lui seul, et sans moyens autres que la légitimité démocratique, gérer le développement du territoire et arbitrer les décisions.}

Peu importe le mode d'organisation municipale, la tendance actuelle au regroupement des municipalités devra faire la distinction entre les fonctions dites de proximité et les fonctions régionales. Dans l'hypothèse où le gouvernement incite les municipalités à se regrouper dans des ensembles plus vastes, comme les MRC par exemple, il devra réserver aux anciennes limites des municipalités et aux lieux-dits des services de proximité qui s'appuient sur le sentiment d'appartenance. Car c'est là que se trouve le terreau porteur des politiques communautaires et familiales. 


\section{La complémentarité rurale-urbaine}

Deuxième question. Comment s'assurer que la nécessaire complémentarité rurale-urbaine se vive de façon harmonieuse, dans le respect et à l'avantage des deux parties? Rappelons que la Loi sur l'aménagement et l'urbanisme avait, en 1980 et pour la première fois, réuni autour de la table de la MRC les municipalités relevant du Code municipal et celles relevant de la Loi des cités et villes. On imagine mal, aujourd'hui, les changements de mentalité que cette opération sous-entendait, il y a seulement 25 ans. Le maire d'une ville de la Côte-de-Sud a mis cinq ans avant de se résoudre à venir s'asseoir à la table de la MRC avec ses collègues ruraux. De tels comportements font aujourd'hui sourire, mais ne sont pas entièrement éradiqués des pratiques rurales-urbaines.

Le modèle de la ville-MRC et de la MRC villes-pôles devrait contribuer à rapprocher encore davantage ces deux mondes. L'un ne devra pas souffrir de l'autre. La nouvelle Loi sur l'aménagement et l'urbanisme devra inscrire en lettres d'or les principes et les règles qui régiront les rapports entre les habitants des villes et des campagnes.

\section{On n'y échappera pas; il faut reprendre le train des réformes.}

\section{L'impôt foncier}

Troisième question. L'impôt foncier ne satisfait plus les besoins grandissants des municipalités. Et pas seulement pour Montréal! Il faudra absolument régler la question des sources de revenu du monde municipal. Les nouvelles responsabilités qui sont accordées aux municipalités ne sont pas suffisamment pourvues des ressources financières adéquates. Pour ce qui est de l'impôt foncier, pourquoi ne serait-il prélevé par le ministère du Revenu qui le redistribuerait ensuite aux MRC villes-pôles et aux villes-MRC ? (À moins de confier un pouvoir de taxation à la MRC, mais ça, c'est une autre histoire; les municipalités et les villes s'y objecteraient. Pourtant, les Communautés de com- munes, en France, sont dotées d'un pouvoir de taxation [une part de la taxe professionnelle] et s'en portent bien.)

Sur la Côte-de-Beaupré, par exemple, si l'impôt foncier était prélevé par le ministère du Revenu, la tentation toute naturelle d'étendre le périmètre d'urbanisation pour récolter de meilleurs revenus ne constituerait plus l'assise financière du développement d'une municipalité. Les revenus de la ville-MRC ou de la MRC villes-pôles seraient attribués en fonction d'objectifs et de critères propres à chaque territoire et définis par les élus municipaux.

\section{Le mieux et le pire}

La responsabilité ultime de l'aménagement du territoire est affaire d'État et c'est à lui que revient le devoir de corriger les iniquités et de favoriser des rapports harmonieux entre les communautés humaines qui habitent les territoires du Québec. Jusqu'ici, les fusions municipales ont plutôt fait battre les gouvernements que de contribuer à les faire élire. Pourtant, une démarche qui exposerait les responsabilités des arrondissements et des conseils de quartier ainsi que les responsabilités régionales dans le cadre de la décentralisation pourrait être gagnante. Et de toute façon, on n'y échappera pas; il faut reprendre le train des réformes.

Restructurer les pouvoirs locaux et régionaux signifie maintenant aller jusqu'au bout de la logique de la décision gouvernementale d'août 1996 de retenir la division territoriale de la MRC comme territoire de développement local et définir les politiques de soutien aux acteurs à partir de ce milieu de vie. Et puisque les acteurs se situent dans les territoires de MRC et dans les arrondissements urbains, c'est là que doivent se trouver les moyens et les institutions de première ligne mis à leur disposition pour stimuler le développement de leur milieu.

Le mieux serait que les élus et les citoyens participent à ce partage des pouvoirs sur leur territoire et rendent la tâche plus facile à l'État qui devra choisir. Le pire serait de continuer de reporter l'échéance. 


\section{Publicité}

\section{Organisations et territoires - Site Web}

